\title{
Optimization of fatigue damage indication in ferromagnetic low carbon steel
}

\author{
Ivan TOMÁŠă , Ondřej KOVÁŘíK ${ }^{\mathrm{b}}$, Jana KADLECOVÁa ${ }^{\mathrm{a}}$, Gábor VÉRTESYc \\ $a_{\text {Institute of Physics ASCR, Prague, Czech Republic }}$

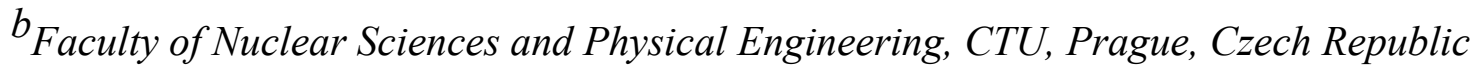 \\ ${ }^{c}$ Institute of Technical Physics and Materials Science, Budapest, Hungary
}

\begin{abstract}
Fatigue damage was investigated by the method of Magnetic adaptive testing (MAT), which is based on systematic measurement and evaluation of minor magnetic hysteresis loops. A large number of magnetic measurements were performed on a single reference series of low carbon steel flat samples, which were fatigued by cyclic bending in an identical way, up to an increasing level of the fatigue damage. The measurements of the magnetic properties of these samples were repeated under varied conditions, including speed of magnetization of the samples, sample temperature during the measurement, choice of the evaluated signal, frequency of the voltage sampling, and range of the applied amplitudes of the magnetizing field/current. Special attention was turned on influence of the thickness of non-ferromagnetic spacers positioned between surface of the samples and flat fronts of the attached magnetizing yokes. On one hand, the spacers decrease values of the induced signal and its derivatives, but on the other hand they substantially increase reproducibility of the measurement and positively influence shapes of the resulting degradation curves. Optimum conditions for the magnetic measurement of the fatigue damage were searched, found, and recommended. The results indicate reliable applicability of MAT to detect early stages of the material fatigue, and to predict its residual lifetime.
\end{abstract}

Keywords: fatigue, residual lifetime, magnetic nondestructive evaluation, ferromagnetic construction materials

\section{Introduction}

The majority of industrial components failures take place due to material fatigue that frequently occurs without early enough warning. Many ferromagnetic components are subjected to alternating load in service, which often causes their structural failure as a result of fatigue [1-3]. Evidently, any improvement of regular, economic and timely nondestructive monitoring of fatigue damage of critical parts of industrial constructions makes an important, actual and challenging issue. In order to check on the health of the construction materials, it is necessary to be able to detect the initiation and the early stages of propagation of fatigue cracks, and to predict residual lifetime of the constructions with a satisfactory earliness of the forecast.

Magnetic measurements are frequently used for characterization of changes in ferromagnetic materials, because magnetization processes are closely related to their microstructure. This makes the magnetic approach an obvious candidate for non-destructive testing, for detection and characterization of any defects in materials and in products made of such materials [4]. Magnetic nondestructive evaluation of fatigue damage of ferromagnetic 
steels was reported in [5]. One of the most frequently and successfully used methods is the traditional hysteresis method. A number of techniques have been suggested, developed and currently used in industry, see e.g. [6, 7]. They are mostly based on detection of structural variations via the classical parameters of major hysteresis loops.

Our recent paper [8] presented principles of a new way of the fatigue monitoring, which was based on application of the Magnetic Adaptive Testing (MAT) method [9]. As MAT relies on generalized magnetic hysteresis properties of the tested materials, it is evident, that the presented way of the MAT fatigue (MATFAT) detection and of the MATFAT residual lifetime monitoring is applicable to ferromagnetic construction materials only. However, ferromagnetic steel and cast iron are so widely used construction materials, that this material limitation can hardly be considered as a serious restraint. In [8], the MATFAT investigation was classified among a number of other magnetic fatigue monitoring methods [10-18] and its advantages and drawbacks were discussed in relation with them. Some very attractive features of the MATFAT investigation were pointed out, its simplicity, reproducibility, reliability and multi-parametric character, in particular.

The main purpose of the present paper is optimization of the MATFAT method for investigation of industrial components with the aim of finding the most sensitive and the most reliable experimental parameters of the measurement. We shall shortly remind the samples shape and materials, details of the applied fatigue test method, and the two used methods of measurement in Section 2, specify the experimental results in Section 3, and discuss the obtained results in Section 4. Section 5 will be devoted to the conclusions of the presented effort.

\section{Samples and Methods of Measurement}

Material of the samples is low carbon steel (S235JR), produced from recrystallizationannealed $\left(550-700^{\circ} \mathrm{C}\right)$ steel sheets, i.e. similar as in [8], but only slightly cold rolled (which resulted in their longer full lifetime, $T$, than in [8]). The fatigue test method and the ways of monitoring the fatigue lifetime applied for the purposes of this paper are identical to those used in our previous work [8] and details, together with explanatory pictures, can be found there. Here we shall summarize the main features only, just in order to make this text as consistent and self-explanatory as possible.

Each flat dog-bone-shaped steel sample, mounted by its bottom head in a vice, was fatigued by resonance cyclic bending (asymmetry ratio $\mathrm{R}=-1$ ) forced by a couple of deflection electromagnets positioned close to the top of the samples, see Fig.1. All the measurements were done at the room temperature of about $20^{\circ} \mathrm{C}$. 


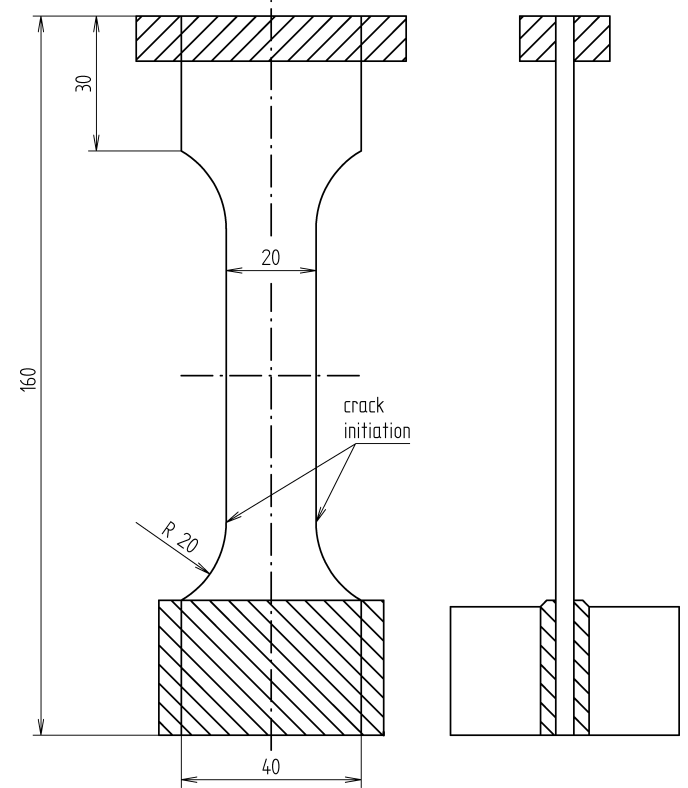

Fig.1 Dimensions and mounting of the flat dog-bone-shaped steel samples (4 mm thick) fatigued by cyclic bending. The right-downwards hatching represents mounting of the bottom head of the sample in non-magnetic jaws of a vice, the right-upwards hatching depicts a ferromagnetic armature at the top end of the sample, which helps to the electromagnetcontrolled cyclic sample deflection by the constant amplitude $\pm 4 \mathrm{~mm}$ and which also settles a convenient resonance frequency of the system close to about $80 \mathrm{~Hz}$.

Cyclic loading of the sample takes place at an adapted SF-Test facility, see e.g. [8, 19], which enables an auxiliary mechanical monitoring of the proceeding fatigue damage via checking the system resonance frequency. The excitation frequency is kept exactly at the resonance frequency of the sample-armature system by maintaining a constant $\pi / 2$ phase-shift between the excitation force of the electromagnets and the sample deflection. During the initial phase of the cyclic loading, hardening or softening of the material influences the specimen stiffness. (Our specimens were actually softened during the initial phase, which decreased the resonance frequency slightly.) Later, during the crack-initiation and the crackpropagation phases, the fatigue cracks effectively reduce the cross-section of the specimen, which results in an accelerated decrease of the resonance frequency. The level of fatigue damage of the samples can thus be reliably estimated from the resonance frequency variation during the fatigue life of the sample [19].

Physical principles, experimental application and ways of evaluation of results of MAT are described in details in the first two chapters of [9]. For the purpose of the present paper, we just point out that MAT proceeds by magnetization of the tested ferromagnetic object by a slowly oscillating magnetic field with a constant absolute value of its rate of change (triangular oscillation) and with amplitude that starts either at a chosen minimum and is increased by a constant step after each period, until magnetic saturation of the sample is approached. Or, alternatively, the amplitude starts at a chosen maximum and is decreased by a constant step after each period, until some chosen minimum is approached. Both approaches are possible, the later saves time, as it does not require artificial demagnetization of the samples before each MAT measurement [19]. Both approaches allow to search all the possible magnetization states of the tested object and to identify parameters of its immediate magnetization, which offers best relation to the investigated property of the sample according to any desired criteria. A voltage signal is induced in a pick-up coil wound around the magnetized circuit. The voltage signal is proportional to the time-derivative of the magnetic 
induction, and because the absolute value of the rate of change of the slowly oscillating magnetizing field is kept constant, it is also proportional to the field-derivative of the magnetic induction, or in other words to the differential permeability, $\mu$, of the magnetized circuit, a part of which is formed by the investigated object. The recorded data thus constitute a large family of minor loops of differential permeability, $\mu$, of the magnetized circuit. The data are processed and interpolated into a large set of descriptors, $\mu(I, A, t)$, of the magnetic state of the magnetized circuit, each of them indexed by a triplet of parameters. They are the actual value of the magnetizing current, $I$, the actual value of the current amplitude, $A$, of the minor loop and the actual value of the fatigue degradation lifetime, $t$, of the sample. (Values of the magnetizing current, $I$, and of the current amplitude, $A$, are used as the practical parameters rather than the corresponding values of the magnetizing field. This can be done as the current oscillations are quasi-static and the field values are proportional to the current ones.) Each sequence of the descriptors, which has the parameters $I=I_{i}$ and $A=A_{j}$ constant and only $t$ is varied from $t=0$ up to $t=T$, and which is normalized by the value of the corresponding descriptor at $t=0$ (i.e. of the 'virgin' or reference sample), is called a normalized degradation function/curve $\mu\left(I_{i}, A_{j}, t\right)$ of the fatigue degradation lifetime, $t$, of the sample. The produced degradation functions/curves are compared with each other and the most sensitive and/or the most indicative of them are picked up for the best indication of the fatigue degradation process. The degradation curves, which were created straight from the induced signal, are usually labeled as the $\mu$-degradation curves.

The complete mapping of the whole set of degradation curves revealed [8], that large values of the magnetizing amplitudes, $A$, (but not at all necessarily the saturation ones) are most convenient for the MAT investigation of fatigue, i.e. for MATFAT.

The "in-situ" MATFAT measurement performed during the fatigue test, was usually carried out in the following way: Always after a chosen number of loading cycles, the test was interrupted, a short solenoid containing both the magnetizing and the pick-up coils was placed over the critical part of the sample, a couple of passive soft magnetic yokes was pressed to the sample (so that they and the sample created a semi-closed magnetic circuit), and one short family of the minor (but rather large) permeability loops was recorded.

Thin nonmagnetic spacers, placed between the yoke faces and the investigated sample, were used to modify quality of the magnetized circuit. After each single MAT measurement was finished, the yokes were taken away and the fatigue test continued for a given next number of loading cycles. Fig.2 shows the sample with the yokes and with the yoke-holder during the magnetic measurement. This approach was used in our previous study [8].

For the optimization of the MATFAT measurement, a different, "ex-situ", method was applied: First, a reference series of virgin samples without any fatigue damage was carefully picked up, so that their magnetic properties were nearly identical. Then, each of them was cycled to a different level of the fatigue damage (see the list in Table 1, below). After that, they were many times measured as individual samples under varied experimental conditions. See the more detailed description in Section 3. 


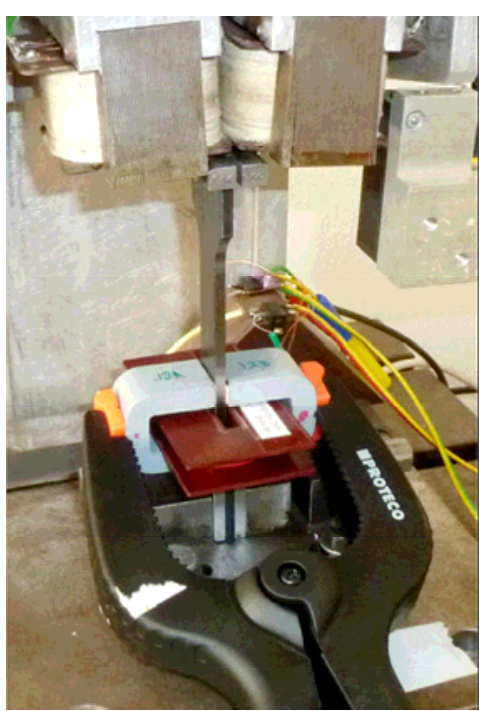

Fig.2 The critical part of the sample is covered by the magnetizing/sensing solenoid and a couple of passive soft yokes pressed to the surface of the sample with a plastic spring holder. The yoke faces are covered with thin nonmagnetic spacers. All is fixed at the bottom in a vice with non-magnetic jaws. The couple of deflection electromagnets can be seen at top of the figure.

\section{Results}

Before the optimum experimental conditions of MATFAT will be here scrutinized, two main ways of the measurement should be mentioned, distinguished and compared, see Fig.3.

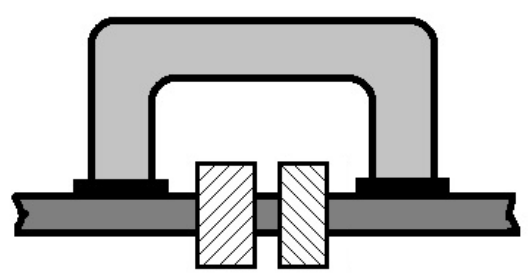

(a)

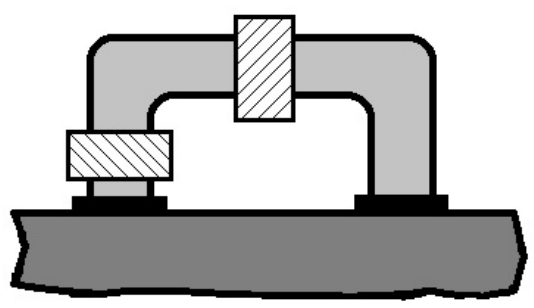

(b)

Fig.3 Geometry of the MATFAT testing with the aid of: (a) passive magnetizing yoke

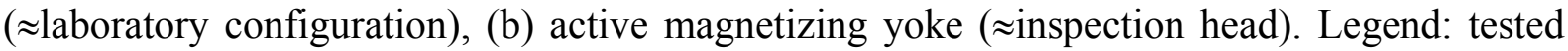
material (dark gray), soft magnetic yoke (light gray), non-magnetic spacer (black), magnetizing coil (right-upwards hatching), pick-up coil (right-downwards hatching).

The fatigued object can be magnetized and sensed by the magnetizing and pick-up coils wound around the body of the object, plus one or even two passive soft magnetic yokes, which help to close artificially the originally open magnetic circuit of the tested body itself. This measuring configuration was applied in great majority of our presented experiments, and for its special geometry of the tested object it can be labeled as the "laboratory" configuration, see Fig.2.

In other, probably more practical cases, when the tested (industrial) object has a shape where the wound-around coils cannot be applied, the tested object can be magnetized and sensed by the magnetizing and pick-up coils wound on a soft magnetic yoke, nicknamed an 
inspection head, and the inspection head is then firmly attached to a geometrically corresponding surface of the tested object.

Our tests showed that MATFAT measurement carried out either by the laboratory configuration or by the inspection head, see Fig.3, on the same series of step-by-step fatigued samples, yield qualitatively identical results, even though laboratory configuration gives usually slightly more sensitive results than the measurement with an equivalent inspection head.

The main purpose of this paper, however, is to specify the principal experimental conditions of MATFAT and to suggest their optimum choice.

For this purpose we shall keep the material, shape, and dimensions of the samples, as well as parameters of the fatigue loading, constant. All those constant properties were described and specified in this text above.

However, we shall challenge the speed of magnetization, the temperature during the experiment, the choice of the evaluated signal, the frequency of voltage sampling + their averaging, the range of the applied amplitudes of the magnetizing field/current, and especially the application, importance, influences and thickness of the non-magnetic spacers, which are positioned between the investigated fatigued specimens surface and faces of the attached yokes.

The measurements, which were carried out and which (because of their straightforward nature and unambiguous outcome) lead towards simple results, will not be described here in much detail. For them we can simply state the following:

The speed of magnetization should be as low as possible in order to prevent substantial influence of the unwanted dynamics of the specimen's domain structure and the unwanted self-induction in the applied coils. On the other hand it must be fast enough to ensure high enough level of the signal induced in the pick-up coil and to make the whole measurement reasonably fast. With the used experimental arrangement the speed of the applied magnetizing current/field about $1 \mathrm{~A} / \mathrm{s}$ (approximately $\leq 5 \mathrm{kA} / \mathrm{m} / \mathrm{s}$ ) gave good results, but application of 3 times larger or 3 times smaller speed was still acceptable. The applied value of the speed of magnetization does not represent any very strict experimental condition. For detailed analysis of the speed of magnetization see [21].

The frequency of voltage sampling is also an important experimental parameter and it must be chosen in such a way, as to correspond to detailed geometry of the measured induced signal. Its proper choice also depends on the applied speed of magnetization, of course. Frequency of sampling of $30 \mathrm{kHz}$ was very satisfactory in our experiments and continuous averaging over 300 voltage samples (digital filtering on-the-fly) proved to be very helpful for keeping the experimental noise at a very acceptable level.

The temperature during the measurement was kept at the room level $\left(\approx 20{ }^{\circ} \mathrm{C}\right)$. Magnetic properties of the used material do not change very fast with temperature at that temperature range, see also [22]. However, if the fatiguing takes place "continuously" in a long series of fast repeating cycles, the material can be warmed up by the applied deformations and it is recommended to wait for the system to cool down to the starting temperature again, before the magnetic measurement is performed. (It is necessary also to keep under control temperature of the magnetizing coil during the very MAT measurement, especially if the coil wire crosssection is not very large.)

The choice of the range of the applied amplitudes of the magnetizing field/current and the choice of the shape of the evaluated signal was discussed enough in our last paper, where both the items were demonstrated graphically, see Figs.6-8 in [8]. The experience suggested the investigated samples should be magnetized by rather large amplitudes of the magnetizing field/current, namely so that the "knees" of the corresponding hysteresis loop are included. Saturation of the samples, however, is not at all necessary. As for the well recognizable 
variations of the signal curves with the increasing fatigue damage, the first derivative of the differential permeability (i.e. the first derivative with respect to the magnetizing field/curent of the voltage signal induced in the pick-up coil) shows very well applicable local (i.e. for some specific ranges of the actual magnitudes of the applied magnetizing current values, $I_{i}$ ) variations and proved to be optimum.

The last but certainly far from least of the experimental conditions, the influence of which should be presented and discussed here, is thickness of the nonmagnetic spacers between the investigated sample surface and the soft-magnetic yoke. The very first idea of inserting the non-magnetic spacers between the sample and the yoke was presented in [23], where the spacers were introduced mainly as a means for decreasing relative fluctuation of the quality of the magnetic contact between the sample surface and the yoke, when the yokes were repeatedly taken away and then attached to the sample surface again. Application of the nonmagnetic spacers proved to be very successful in this.

However, the nonmagnetic spacers influence not only the level of the magnetic contact fluctuation, but they also decrease values of the induced voltage signals and - what is especially important at MATFAT - they also modify shapes of the signals and of their derivatives. The presented experiments, see Figs.4-6, illustrate influence of the nonmagnetic spacers with various thickness values $S p=0,1 d, 2 d, 3 d, 4 d(d=0.045 \mathrm{~mm})$ on the signals, $\mu(I)$, on the first derivative of the signal, $\mu^{\prime}(I)$, and on the final results of MATFAT. The described series of experiments are performed on a reference series of samples "Rxx", where xx means percentage of the spent fatigue lifetime, $t$, i.e. the ratio of the applied number of loading cycles to the expected total number, $T$, of loading cycles to failure. The Rxx samples were carefully picked up from a large set of the commercial steel samples so, that the virgin (i.e. not-fatigues) signal of all the seven samples was identical. After this choice of samples was done, each of the seven originally identical samples was fatigued down to a different level of the fatigue damage. The fatigue damage values are given in Table 1. The experimentally measured nominal total lifetime of the Rxx samples (in kilocycles of the fatigue bending periods) is $T=560 \mathrm{kc}$.

Table 1

\begin{tabular}{|c|c|c|}
\hline Sample & $\begin{array}{c}\text { Percentage of } \\
\text { spent lifetime } \\
{[\%]}\end{array}$ & $\begin{array}{c}\text { No of fatigue } \\
\text { bending periods } \\
{[\mathrm{kc}]}\end{array}$ \\
\hline R00 & $0 \%$ & $0 \mathrm{kc}$ \\
\hline R05 & $5 \%$ & $30 \mathrm{kc}$ \\
\hline R25 & $25 \%$ & $140 \mathrm{kc}$ \\
\hline R63 & $63 \%$ & $350 \mathrm{kc}$ \\
\hline R80 & $80 \%$ & $446 \mathrm{kc}$ \\
\hline R89 & $89 \%$ & $498 \mathrm{kc}$ \\
\hline R96 & $96 \%$ & $538 \mathrm{kc}$ \\
\hline
\end{tabular}

During the spacer thickness optimization the experimental conditions of MATFAT measurements on the reference series of samples, Rxx, were identical, with the exception of the spacer thickness. The laboratory configuration (see Fig.3) with two identical passive yokes (two transformer C-yokes with cross-section $10 \times 25 \mathrm{~mm}^{2}$, inner distance between the legs $15 \mathrm{~mm}$ and outside height in the bow $36 \mathrm{~mm}$ ) was used. The temperature was $20{ }^{\circ} \mathrm{C}$, the speed of magnetization was $1 \mathrm{~A} / \mathrm{s}(\leq 5 \mathrm{kA} / \mathrm{m} / \mathrm{s})$, the maximum magnetizing current amplitude was $\pm 1.7 \mathrm{~A}$, the number of magnetizing coil windings, $N=200$, the number of pick-up coil windings, $n=100$, frequency of the voltage sampling was $30 \mathrm{kHz}$, and the number of averaged 
voltage samplings was 300 . Fig. 4 shows comparison of the R00 sample (virgin) $\mu(I)$-curves and of the corresponding $\mu^{\prime}(I)$-curves for the spacers with different thickness values $S p=0,1 d$, $2 d, 3 d, 4 d(d=0.045 \mathrm{~mm})$.

The different spacer thickness was realized by gluing up to four layers of a $0.045 \mathrm{~mm}$ thick scotch tape to the fronts of the yokes.

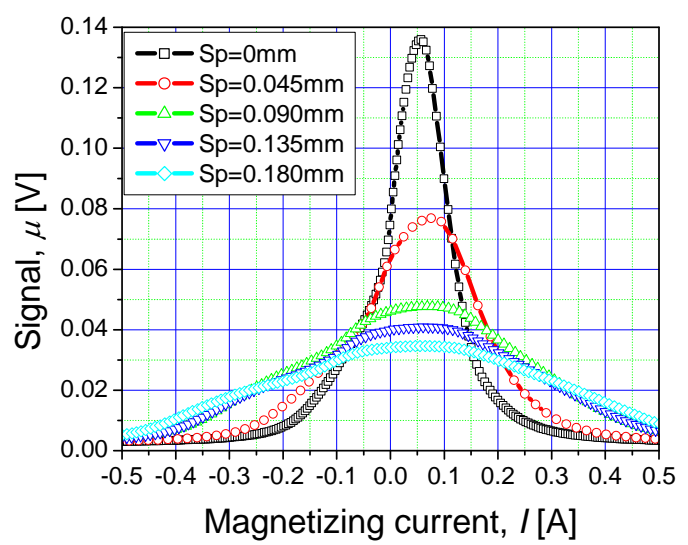

(a)

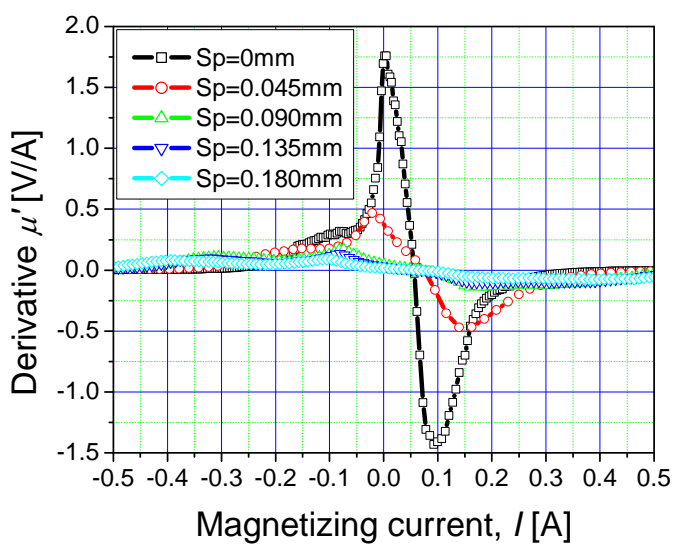

(b)

Fig.4 (a) The virgin directly induced voltage $\mu(I)$-signals, (b) the first derivative $\mu$ '(I) of the signals of the R00 sample for the spacer thickness values $S p=0,1 d, 2 d, 3 d, 4 d(d=0.045 \mathrm{~mm})$. Only the part of the signals corresponding to the "increasing" section of the hysteresis loop is shown for each experimental configuration.

As pointed out earlier, the $\mu^{\prime}(I)$-curves show a distinct variation with the fatiguedamage of the samples at several ranges of $I$. Fig.5 presents different evolutions of the $\mu^{\prime}(I)$ curves for the whole reference series Rxx, when the different spacers $S p=0, \quad, 4 d$ are used.
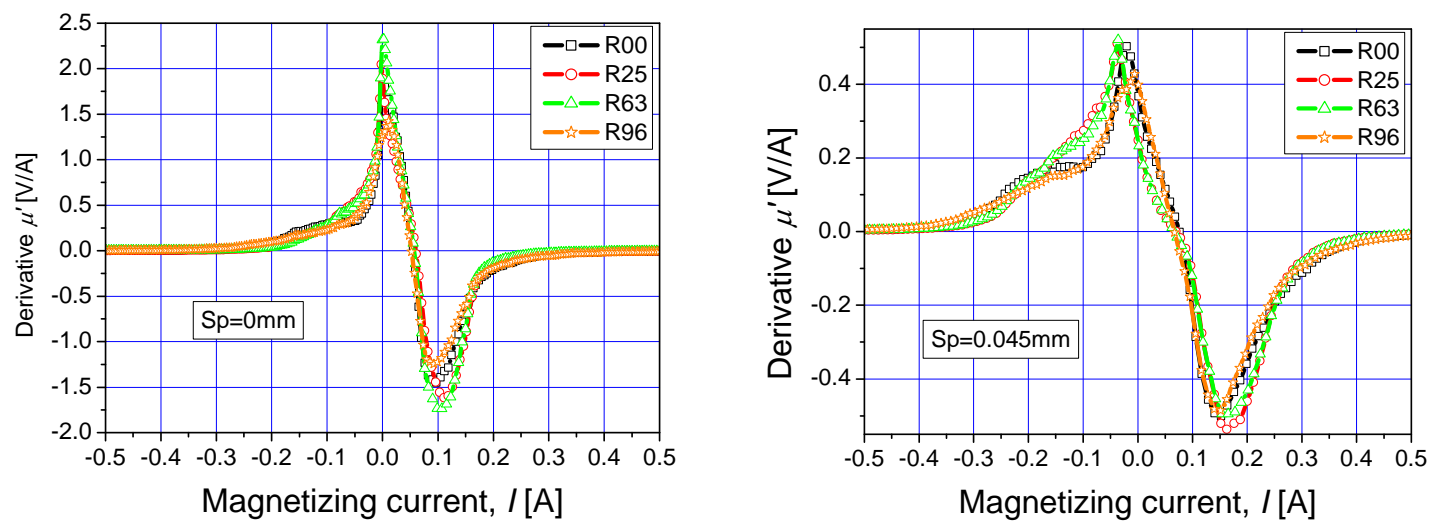

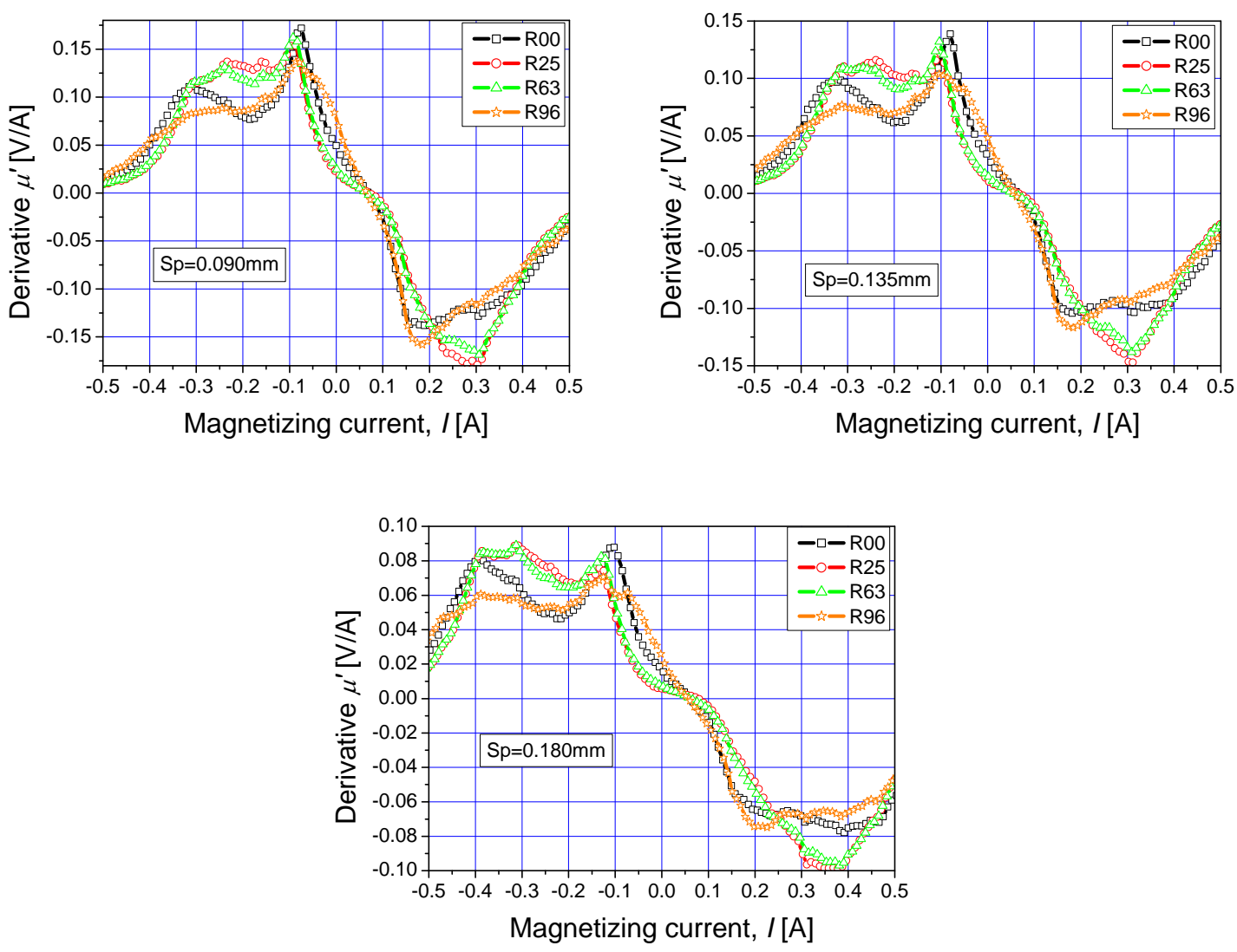

Fig.5 Evaluation of the $\mu$ '(I)-curves with the increased fatigue damage for different thickness values of the applied spacers $S p=0,1 d, 2 d, 3 d, 4 d(d=0.045 \mathrm{~mm})$. Only curves for samples R00, R25, R63 and R96 are presented here. Note the very different vertical scales of the plots.

Mutual comparison of actual values of the $\mu^{\prime}(I)$-curves for chosen magnitudes of the applied magnetizing current values, $I_{i}$, allows to draw the $\mu^{\prime}(t)$-degradation curves for each of the applied thickness of the spacer. Fig. 6 shows shapes of some of the $\mu^{\prime}(t)$-degradation curves (of those, chosen by the experimenter for their convenient shapes, which clearly reflect the process of the samples degradation) for all the used spacer thickness for all measured samples.
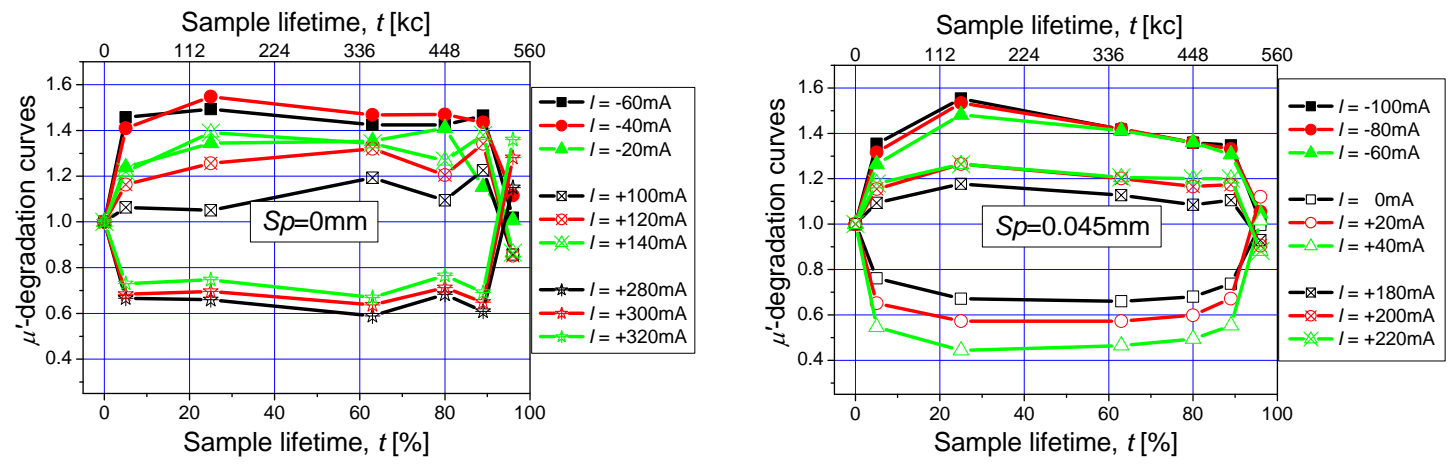

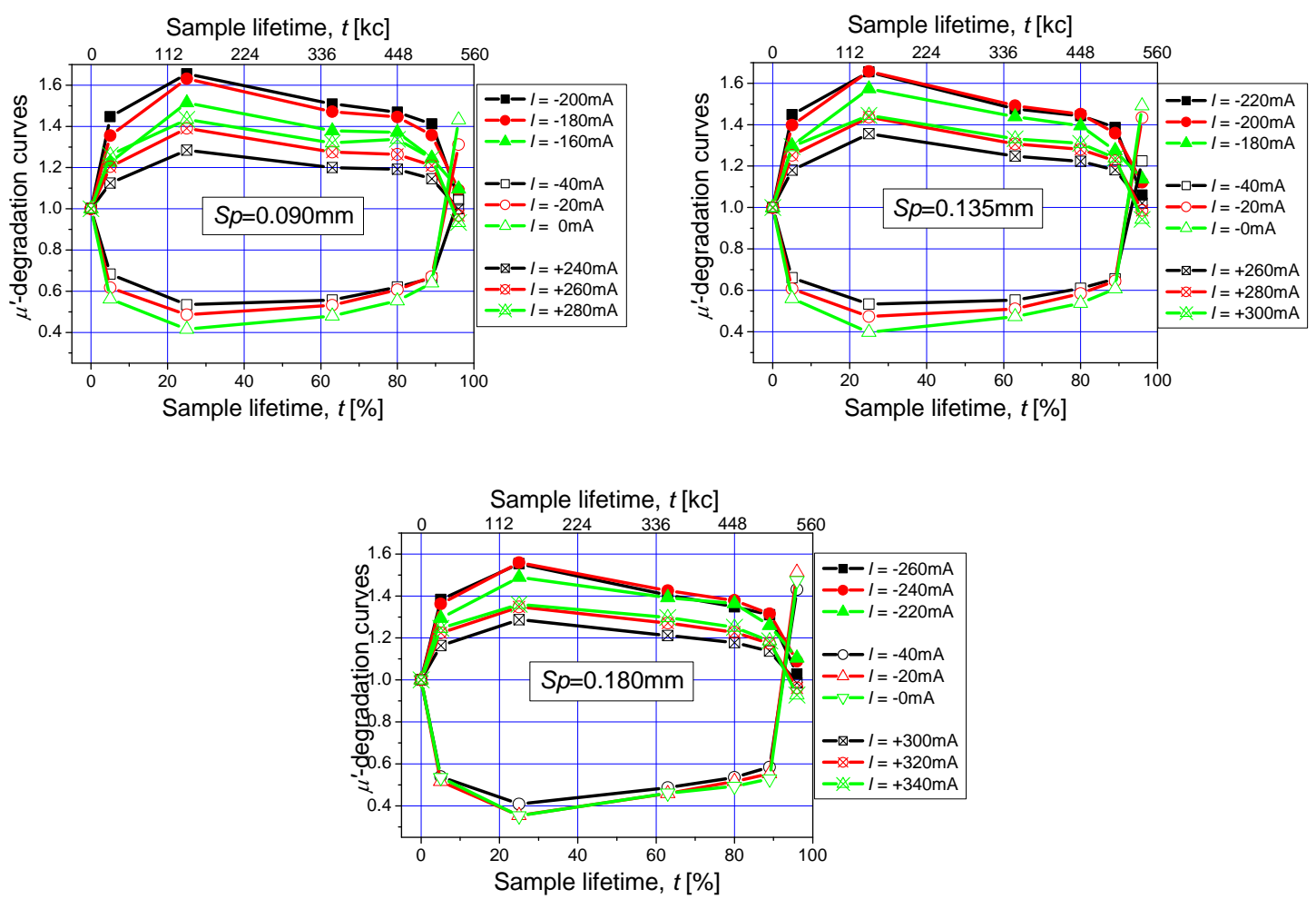

Fig.6 The experimenter's choice of several resulting $\mu^{\prime}(t)$-degradation curves (some of those, reflecting clearly the process of the samples degradation) for the spacer thickness values $S p=0,1 d, 2 d, 3 d, 4 d(d=0.045 \mathrm{~mm})$. Note the identical vertical scales of all the plots. Also note that similarly looking $\mu^{\prime}(t)$-degradation curves are generally obtained for different magnetizing current values, $I_{i}$, if the spacer thickness values are different. Relative error of the points in the plot for $S p=0$ is about $\pm 10 \%$. In all the other plots, however, the relative error does not exceed $\pm 2 \%$, which is hardly the size of any of the point-symbols. Current amplitude of the minor loops at the measurements of all the presented degradation curves was $A= \pm 1700 \mathrm{~mA}$.

\section{Discussion}

All experiments described in this paper were realized on flat steel samples shaped as shown in Fig.1, fatigued by cyclic bending, as described in Section 2. Application of just this way of fatiguing of flat-shaped samples has three important points of advantage. First it can utilize - next to the here still experimentally rather tested than routinely used MATFAT - also the resonance frequency course provided by the well established SF-Test method, and get thus the direct mechanical information about the actual level of the fatigue damage. Second, the resonance frequency of the cyclic bending can be rather high (about $80 \mathrm{~Hz}$ in our experiments), which makes the time necessary for a complete fatigue destruction of the samples by about an order of magnitude shorter than the necessary time for similar comparable number of cycles in any hydro-mechanical uniaxial push-pull fatigue system. And third, the flat shape of the samples makes it easy to attach simple magnetizing yokes firmly and with a good magnetic contact to the flat surfaces of the samples. The yokes - either passive or active - make it possible to close artificially the originally open magnetic circuit.

Without the yokes (but for rare special cases of tested objects having a shape of a magnetically closed circuit) it would be impossible to magnetize the tested object enough (with the relatively small and short magnetizing coils, which can be placed on the object or on 
the yoke) for the application of MATFAT. Application of the yokes simulates a closed circuit. The circuit is not uniform, however. Evidently, the material of the applied yokes is generally different from the tested object, but the major non-uniformity of the magnetic circuit is brought about by the unavoidable air gaps between the yoke fronts and the surface of the tested object. Even the best-polished surfaces do not yield an ideal magnetic contact and what is even worse, every little dirt or small distortion of the matching surfaces influences quality of the magnetic contact. By the general experience, reproducibility of quality of the magnetic contact is low, and consequently the scatter of measured MAT results at repeated removing and attaching of the same yoke at the same place of the object surface is high.

It was shown in [23], that reproducibility of such repeated MAT measurement could be substantially increased if the yoke and the object are not attached to each other directly but if a small gap is intentionally created between the two surfaces. The easiest way of creating a constant small gap is to insert a thin non-ferromagnetic spacer in the place. The convenient spacer decreases the unwanted fluctuation of the MAT results, decreases also the induced signal (which is sometimes not very much welcomed, so that careful balance must be found), and also modifies shape of the induced signal and of its first derivative, which proved to be rather welcomed at MATFAT. Fig.4 and especially the series of plots in Fig.5 show the substantial modifications of both the height and the shape of the $\mu^{\prime}(I)$-curves with thickness values of the applied spacers $S p=0,1 d, 2 d, 3 d, 4 d$. The considerable reduction of the $\mu^{\prime}(I)$ height looks formidably for outlooks of the final evaluation of the $\mu^{\prime}(t)$-curves. However, probably due to the simultaneous sizeable decrease of fluctuation of the $\mu^{\prime}(t)$-curves, the final $\mu^{\prime}(t)$-curves are equally reliable with thicker spacers - if not more - than those with the very thin one, not speaking about the MATFAT measurement without any spacer. The best illustration of this fact can be seen in the plots of Fig.6. Actually shapes of the final $\mu^{\prime}(t)$ degradation curves with $S p=2 d, 3 d$, and $4 d$ look mutually equivalent both by their sensitivity and by their scatter. Application of even thicker spacers starts to decrease sensitivity of the $\mu^{\prime}(t)$-degradation curves. Usable $\mu^{\prime}(t)$-degradation curves were obtained even with spacers $1 \mathrm{~mm}$ thick, however. Influence of the spacer thickness on the $\mu^{\prime}(t)$-degradation curves is mediated by the fact that often similarly looking curves are obtained at different values of the magnetizing field/current, $I_{i}$, at different values of $S p$, see Fig.6c, d, e.

The slow oscillations of the current applied to the magnetizing coil are triangular, with an amplitude, which was determined by the foregoing analysis of MAT (not within this very paper, here it is just stated, but see [8] for details). Shape of the magnetizing current oscillations is given by the used function generator. As the time rate of change of the current is low, usually practically quasi-static, also the magnetizing field can be considered triangular, if the magnetic circuit is uniform (e.g. a ring-shaped sample). This was checked up experimentally. If the magnetic circuit is not uniform (e.g. for a magnetic circuit including the sample and the flux-closing yoke, as it is in our present case), the triangular shape of the field is not apparent, but as the signals recorded on the ring samples and on the sample-yoke circuits differ mainly by their amplitude, but do not differ substantially by their shape (this was many times checked up experimentally), we are sure, the magnetizing field is close to the triangular shape itself, even in the sample-yoke circuits. Deformations of the field shape from the triangular one, in the case of the sample-yokes circuits (especially with thicker nonmagnetic spacers), can have the consequence, that the $\mu$ - and the $\mu$ '-degradation curves applied in MATFAT do not correspond exactly to the physical entity called "permeability". However, this is not a crucial problem within MATFAT, and the notation is kept in spite of this recognized fact, for the sake of simplicity of description. The point is, that the degradation curves reflect the process of the material fatigue degradation, which is the main point within MATFAT. 


\section{Conclusions}

A large number of MATFAT measurements were performed on a single reference series of low carbon steel flat samples, which were fatigued by cyclic bending in an identical way, up to an increasing level of the fatigue damage. The measurements of the reference samples were repeated under varied conditions of the experiment, including speed of magnetization of the samples, temperature during the experiment, choice of the evaluated signal, frequency of the voltage sampling + their averaging, and range of the applied amplitudes of the magnetizing field/current.

Special attention was turned on influence of thickness of non-ferromagnetic spacers positioned between surface of the samples and flat fronts of the attached magnetizing yokes. The spacers decrease magnitude of the magnetic field inside the samples and through this also decrease values of the induced signal and its derivatives (which is the principal indicator of the fatigue damage level at MATFAT). The spacers, however, substantially increase reproducibility of the measurement. Taken all their influence together, spacers about $0.1 \div 0.2 \mathrm{~mm}$ thick improve general sensitivity of the resulting $\mu^{\prime}(t)$-degradation curves and are strongly recommended to be used. Even thicker spacers - if for any reason necessary can be successfully applicable, but sensitivity of the final $\mu^{\prime}(t)$-degradation curves is then lowered.

\section{Acknowledgement}

The authors of this paper were financially supported by project No.14-36566G of the Czech Science Foundation, but for G.V. who was supported by Hungarian Scientific Research Fund (project K 111662) and by JSPS and HAS under the Japan-Hungary Research Cooperative Program.

\section{References}

[1] Pesicka J, Kuzel R, Dronhofer A, Eggeler G 2003 The evolution of dislocation density during heat treatment and creep of tempered martensite ferrite steels, Acta Mater. 51 (2003) 4847-4862

[2] Nian Li, Baiping Du 1995 The effect of low-stress high-cycle fatigue on the microstructure and fatigue threshold of a 40Cr steel, Int. J. Fatigue 17 (1995) 43-48

[3] Xiulin Z 2001 On some basic problems of fatigue research in engineering, Int. J. Fatigue 23 (2001) 751-766

[4] Jiles DC 2001, Magnetic methods in nondestructive testing, K.H.J. Buschow et al., Ed., Encyclopedia of Materials Science and Technology, Elsevier Press, Oxford, p.6021

[5] Gilanyi A, Morishita K, Sukegawa T, Vesaka M, Miya K 1998 Magnetic nondestructive evaluation of fatigue damage of ferromagnetic steels for nuclear fusion energy systems, Fusion Eng. Des. 42 (1998) 485-491

[6] Jiles DC 1988, Review of magnetic methods for nondestructive evaluation, NDT International 21311.

[7] Blitz J 1991, Electrical and magnetic methods of nondestructive testing, Bristol, Adam Hilger IOP Publishing, Ltd.

[8] Tomáš I, Kovárík O, Vértesy G and Kadlecová J 2014 Nondestructive indication of fatigue damage and residual lifetime in ferromagnetic construction materials Meas. Sci. Technol. 25 (2014) 065601

[9] Tomáš I and Vértesy G 2012 Magnetic adaptive testing: Nondestructive Testing Methods and New Applications ed M Omar (Rijeka: InTech) ISBN: 978-953-51-0108-6 www.intechopen.com/articles/show/title/magneticadaptive-testing 
[10] Dobmann G, Debarberis L and Coste J-F 2001 Aging material evaluation and studies by non-destructive techniques (AMESNDT) - a European network project Nucl. Eng. Des. 206 363-74

[11] Sagar S P et al 2005 Magnetic Barkhausen emission to evaluate fatigue damage in a low carbon structural steel Int. J. Fatigue 27317

[12] Lo C C H et al 1999 Monitoring fatigue damage in materials using magnetic measurement techniques J. Appl. Phys. 85 4595-7

[13] Doubov A A 1997 A study of metal properties using the method of magnetic memory Met. Sci. Heat Treat. 39 401-2

[14] Chen Z J et al 1994 Estimation of fatigue exposure from magnetic coercivity J. Appl. Phys. 756975

[15] Lo C C H, Tang F, Jiles D C and Biner S B 1999 Evaluation of fatigue damage using a magnetic measurement technique IEEE Trans. Magn. 353977

[16] Bezlyudko G Y, Yolkina Y I, Solomakha R N and Popov B Y 2010 Coercivity metering as the basic method of a non-destructive testing of fatigue and as the priority method in the diagnostic set ECNDT: European Conf. and Exhibition on Non-destructive Testing (Moscow)

[17] Devine M K, Kaminski D A, Sipahi L B and Jiles D C 1992 Detection of fatigue in structural steels by magnetic property measurements J. Mat. Eng. Perf. 1 249-53

[18] Lo C C H, Tang F, Biner S B and Jiles D C 2000 Effects of fatigue-induced changes in microstructure and stress on domain structure and magnetic properties of $\mathrm{Fe}-\mathrm{C}$ alloys J. Appl. Phys. 876520

[19] Kováŕík O, Siegl J and Procházka Z 2008 Fatigue behavior of bodies with thermally sprayed metallic and ceramic deposits J. Therm. Spray Technol. 17 525-32

[20] Ušák E, private communication

[21] Tomáš I, Vértesy G and Kadlecová J 2009 Influence of rate of change of magnetization processes on sensitivity of Magnetic Adaptive Testing J.Magn.Magn.Mater. 321 (2009) 1019-1024

[22] Vértesy G, Uchimoto T, Tomáš I and Takagi T 2010 Temperature dependence of magnetic descriptors of Magnetic Adaptive Testing

IEEE Trans.Magn. 46 (2010) 509-512

[23] Tomáš I, Kadlecová J and Vértesy G 2012 Measurement of flat samples with rough surfaces by magnetic adaptive testing IEEE Trans. Magn. 48 1441-4 